\title{
Early Warnings for Drought in Europe A Multihazard System
}

\author{
Adapted from "Potential \\ of pan-European Seasonal \\ Hydro-Meteorological Drought \\ Forecasts Obtained from a \\ MultiHazard Early Warning \\ System," by Samuel Jonson \\ Sutanto (Wageningen University \\ and Research), Henny A. J. Van \\ Lanen, Fredrik Wetterhall, and \\ Xavier Llort. Published online in \\ $B A M S$, April 2020. For the full, \\ citable article, see DOI:10.1175 \\ /BAMS-D-18-0196.2.
}

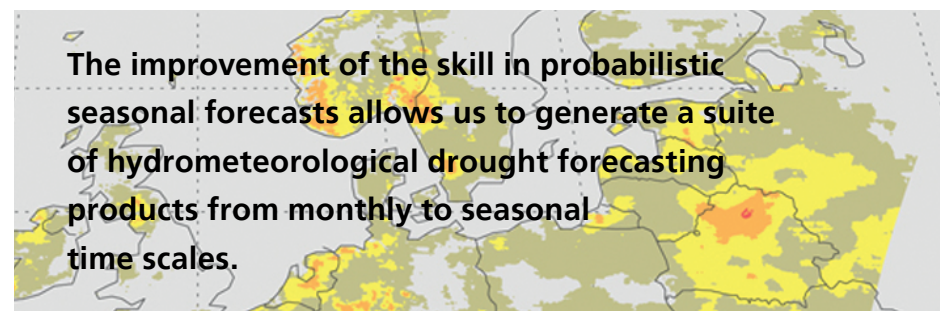

o meet the targets of the United Nations Sendai Framework for Disaster Risk Reduction 2015-30, multihazard early warning systems (EWS) are needed. The weather-induced EWS that have been developed, however, are mostly designed to predict high-flow extremes like floods and flash floods, landslides, and storm surge. While these EWS require shorter lead forecasts, over hours and days, a drought EWS requires monthly to seasonal forecasts. The historical inability to produce robust monthly precipitation forecasts is the main reason why seasonal drought EWS are less developed than many short-time-scale hazard warning systems.

Recently, the use of probabilistic seasonal weather forecasting systems based on numerical weather prediction models has become more common, since the skill of these models has greatly improved. This has led to the development of operational seasonal drought EWS in some regions, such as the United States and Africa. Those EWS mainly use precipitation, temperature, and soil moisture forecast data. In the United States, operational real-time drought monitoring provides information on current drought conditions as well as seasonal drought 
forecasts using the standardized precipitation index (SPI)-6, and the anomalies of precipitation, temperature, soil moisture, and streamflow.

In Europe, the need for seasonal drought EWS has risen as drought occurrences have increased over past decades. One of the costliest European droughts was in 2003, with estimated losses of at least EUR 8.7 billion, while the heat-related death toll reached 70,000 . In the past 30 years, the economic loss due to droughts was estimated to be more than EUR 100 billion. The losses will likely worsen in the future because of climate change and increased water use.

In response to these enormous economic losses, several European countries (e.g., Switzerland and Germany) have developed a drought monitor and warning system. The German drought monitoring system provides daily agricultural drought information based on a soil moisture index (SMI), while the Swiss rely on a system based on soil moisture, runoff, groundwater level, and snow water equivalent on a weekly basis up to three months in advance. At the pan-European level, the EU Joint Research Centre (JRC) has developed the European Drought Observatory (EDO) to provide European-wide information on droughts. In addition to monitoring ongoing droughts, the EDO forecasts the soil moisture anomaly up to 7 days in advance at $5-\mathrm{km}$ spatial resolution with a 2-day delay, and predicts drought (SPI-1) on a monthly basis with a 3-month lead time.

\section{The Multi-Hazard EWS}

To provide operational drought forecasts for Europe with a temporal resolution of more than a week, including all water cycle components, a seasonal drought EWS has been developed under the EU-funded Enhancing Emergency Management and Response to Extreme Weather and Climate Events (ANYWHERE) innovation. The drought EWS has been running in a preoperational mode since mid-2018. The objective of ANYWHERE is to employ innovative technologies to build a pan-European multihazard EWS (MH-EWS) that will address all major weather-related natural hazards in Europe, such as floods, flash floods, debris flows, landslides, storm surges, heat waves, air quality, wildfires, droughts, convective storms, severe winds, and heavy snowfall.

The drought EWS (DEWS) developed under ANYWHERE [ANYWHERE DEWS (AD-EWS)] is a part of the MH-EWS and is complementary to the current pan-European operational system (EDO) by producing probabilistic drought forecasts with lead times up to 7 months. The drought monitoring products of the EDO are also integrated into the MH-EWS. The current AD-EWS is driven by the latest ECMWF seasonal forecast system [SEAS 5 (S5)] and a state-of-the-art hydrological model LISFLOOD on the pan-European scale. The seasonal hydrological forecasts are provided through the European Flood Awareness System (EFAS), part of the Copernicus Emergency Management Services. The AD-EWS generates a comprehensive list of drought indices (standardized drought indices, threshold drought indices, and areal drought indices). For standardized indices, the SPI- $x$, the precipitation evaporation index (SPEI- $x$ ), the runoff index (SRI- $x$ ), and the groundwater index approaches (SGI-1) have been implemented with accumulation periods $x=1,3,6$, and 12 months. The variable threshold method identifies drought events and characteristics such as duration, onset, termination, and deficit volume.

The AD-EWS is different from other operational drought EWS. First, it provides a wide range of seasonal hydrometeorological ensemble drought forecasting products for most water cycle components (precipitation, soil moisture, runoff, groundwater, and discharge). Second, it has a nominal spatial resolution (like EDO) of $5 \mathrm{~km} \times 5 \mathrm{~km}$. Only catchment-scale drought EWS have a higher spatial resolution. The up-to-date drought forecasting produces a monthly comprehensive suite of forecasting products and drought identification approaches at a high resolution. The current generation of operational drought EWS uses only the SPI, soil moisture anomaly, runoff anomaly, and streamflow anomaly as drought indices, with a rather coarse spatial resolution of $30 \mathrm{~km}$.

The MH-EWS, as the main platform for the AD-EWS, is a cloud-based platform that integrates the models developed within ANYWHERE and existing pan-European 
platforms; it outputs gridded maps, time series, and bar diagrams.

\section{Examples of AD-EWS drought forecasting products}

The AD-EWS fulfills the needs of different users and covers multifaceted drought phenomena. With the wide range of drought products provided in the AD-EWS, different drought impacts can be investigated. Not every impact can be derived from each drought index, so it is very important to deliver suitable indices for each targeted impact. In the agricultural sector, for instance, water availability is more relevant than runoff. This information can be obtained from, for example, the SPI- $x$, SPEI- $x$, and precipitation and soil moisture threshold products. For water resources managers, on the other hand, the discharge, runoff, and groundwater drought forecasting products (e.g., discharge and runoff threshold indices, SRI- $x$, SGI-1) are far more important than products based on precipitation forecasts (e.g., SPI- $x$, precipitation threshold). Users of AD-EWS can decide which indices best suit their needs.

All drought forecasting products using different standardized indices produce similar drought patterns in Europe. In the severe 2018 drought, for example, northern, central, and northeast European countries and the United Kingdom were forecasted to experience mild to extreme drought events based on SPI-6, SPEI-6, and SRI-6. The forecasted groundwater index (SGI), however, indicated only mild to moderate drought for the same drought regions. All products correctly forecasted drought severity to increase in the second and third month of the event (June and July 2018).

While standardized drought indices products give monthly results, the threshold drought method provides daily information. The forecasted drought onset and termination will inform users about the day the longest drought will start in a certain region and the day when it will end, which is useful for water resources management. The forecasted drought deficit volume/average cumulative deviation from threshold is potentially useful for predicting water deficit for the coming months. Thus, managers can decide to release less water from the
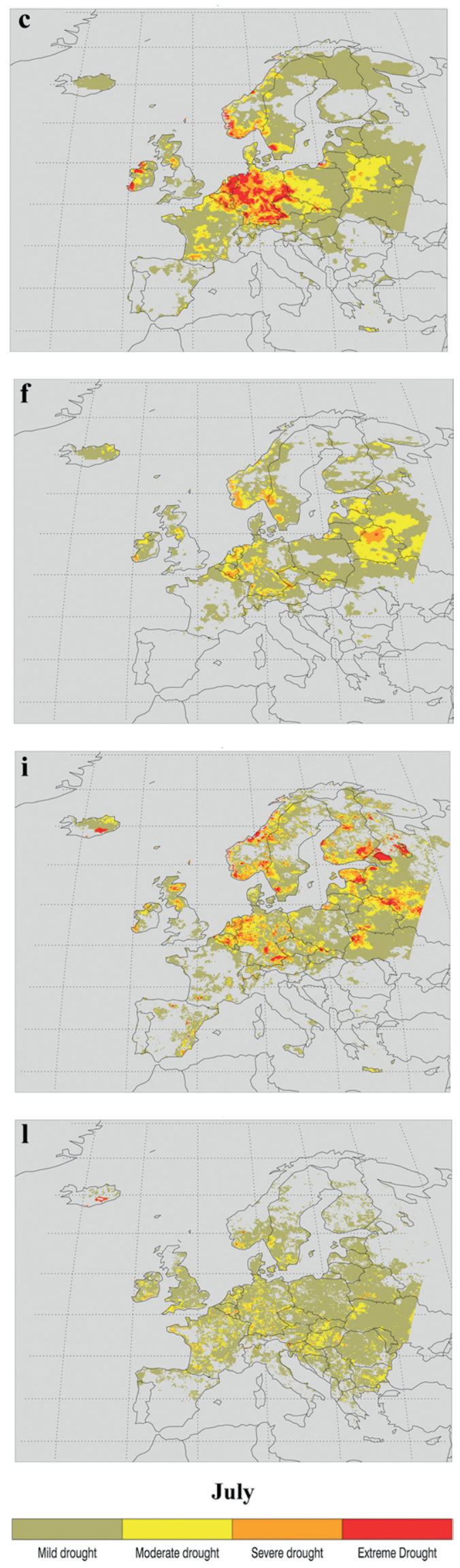

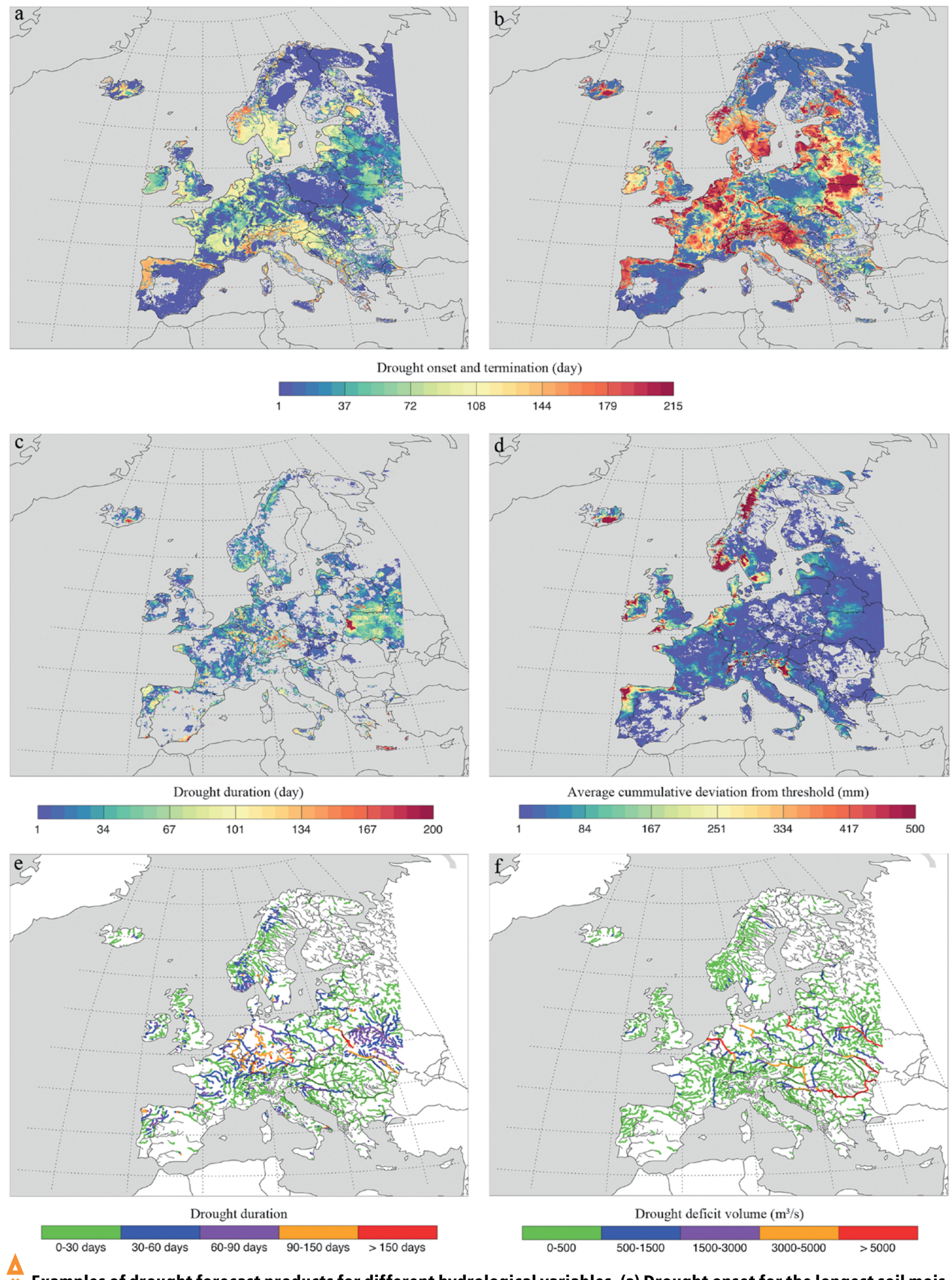

* Examples of drought forecast products for different hydrological variables. (a) Drought onset for the longest soil moisture drought event, (b) drought termination for the longest soil moisture drought event, (c) drought duration in the runoff, (d) average cumulative deviation from the threshold in groundwater, (e) drought duration in discharge, and (f) cumulative drought deficit volume in discharge. All data hold for the median of the 51 ensemble members within the forecast period of 7 months and obtained from the forecast on 2 May 2018. 


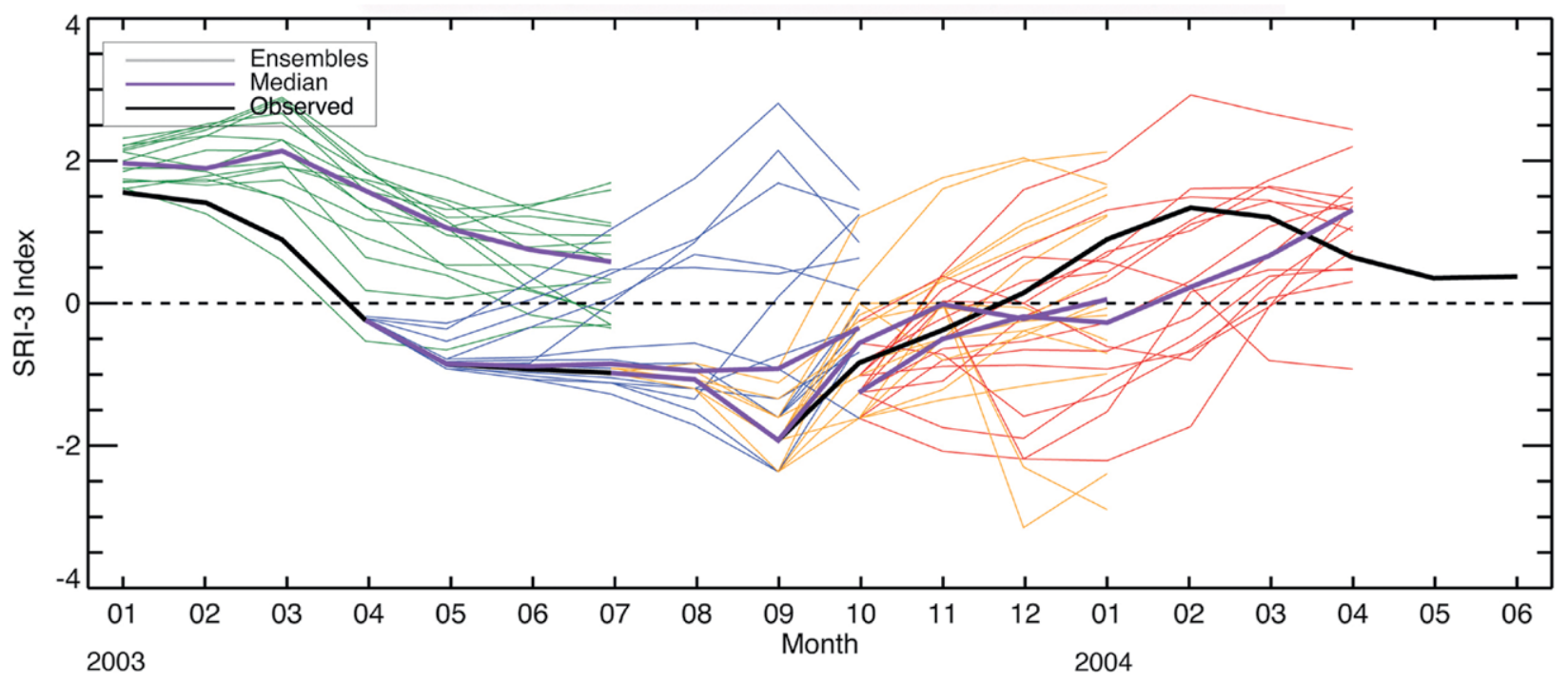

reservoirs, for instance, to maintain ecological minimum flow or restrict groundwater extraction for irrigation. In contrast, the standardized drought indices cannot determine the drought deficit volume.

In addition to pan-European maps with forecasted drought occurrence and characteristics, the AD-EWS also provides areal drought indices, which are time series of the average percentage area in drought for each month over a 7-month forecast. The time series and duration of the drought are forecasted using both the standardized indices and threshold drought indices. The forecasted 7-month drought deficit volume in precipitation of each ensemble member indicates how severe the drought will be over Europe.

\section{The pan-European 2003 drought}

We used the 2003 drought that covered much of Europe to validate the AD-EWS. We selected two indices to represent the meteorological drought (SPI) and hydrological drought (SRI). SPI-3 and SRI-3 forecasted severe to extreme drought in west and central Europe. Observations showed a somewhat larger affected area. To investigate the skill of the forecasts, we present a simple new approach based on a categorical drought classification method. We produce a forecast skill score relatively easily understood by end users. In 58\% of Europe, the forecast score of the meteorological drought is 0 , indicating the forecasted drought class
* Seven-month hydrological (SRI-3) forecasts (median in purple and ensemble members in gray) and SRI-3 derived from proxy observed runoff of the European 2003 drought for one grid cell $(5 \mathrm{~km} \times 5 \mathrm{~km})$ in central France. The 7-month forecast is presented at four issue times: January (green), April (blue), July (orange), and October (red) 2003. is equal to the observed class. In $19 \%$ of the area the score is +1 , meaning the forecast overestimates the observed drought by one class; $20 \%$ of the area has a score of -1 , an underestimation bias. In some European regions (e.g., southeast France), AD-EWS is unable to produce the same drought class as observed (difference up to 4 classes).

In forecasts of meteorological drought (SPI with accumulation periods of 1, 3, 6, and 12 months) for the pan-European domain (for lead times of $1-5$ months), $40 \%-50 \%$ of the area showed perfect forecasts $($ score $=0$ ) for the SPI-1 at all lead times (except SON). For SPI-3, with a lead time of 1 month, the area increases to $50 \%-70 \%$. As expected, the area with perfect scores decreases for longer lead times. SPI-12, which has the longest accumulation period, produces the highest forecasting score, with perfect forecasts for $>60 \%$ of the area up to 3 months ahead in all seasons. Overall, forecast scores for hydrological drought (SRI) are better than for meteorological drought. The area of perfect forecasts for SRI-1 is comparable with that of SPI-3 and SPI-6, while the score for SRI-3 is comparable with that of SPI- 6 and SPI-12. Interestingly, meteorological drought forecasts tend to underestimate the drought while hydrological drought forecasts tend to overestimate it.

In general, the observed hydrological drought event in 2003 is within the ensemble spread. The median forecast initiated in January 2003 produces a higher drought 
index than the observed, and it is closer to the lower ensemble members than to the median. It indicates good skill for forecasts made in April and July 2003. The forecast made in October 2003 showed the drought would be alleviated in 2004, although the forecasted drought is still more severe than the observed drought. The median of 15 ensemble members is better in representing the drought severity than the 25 th percentile.

Even though the AD-EWS can provide different seasonal drought forecasting products with better skill than seasonal weather forecasts, end users want to know how confident they can be with the results. To date, we can correlate the user-defined trust level of our forecasts with the forecast skill itself.

Beyond that general statement, the level of confidence can also be based on the skill of the specific forecast components of greatest importance to each end user. For example, some find that the skill of forecasting drought classes (mild, moderate, severe, and extreme) is most important, while for others, lead time is the most critical forecast component.

\section{Conclusions}

The ability of the AD-EWS to provide diverse hydrometeorological products and operational seasonal drought predictions in high spatial resolution serves a broad range of users' needs in Europe. With this information, end users can take preliminary contingency actions, such as implementing water priority rankings, planning crop irrigation, reducing hydropower, planning cargo for waterborne transport, and conserving water for ecosystems.

\section{$\equiv$ METADATA}

BAMS: What's an essential improvement represented in this drought early warning system?

\section{Samuel Sutanto (Wageningen University and Research): With the wide range of drought products pro- vided in the system, different drought impacts can be investigated. Drought impacts are controlled by different drought indices, so it is very important to deliver targeted indices for each impact. The AD-EWS fulfills the needs of different users and covers the mul- tifaceted drought phenomenon.}

BAMS: How did the idea for such a system originate?

SS: It was started by user's requirements from different countries on drought products. In past international projects, many early warning systems only provided general meteorological drought forecasts. Thus, not all the available products covered all user needs.

BAMS: What got you initially interested in drought forecasting?

SS: Many forecasting studies have focused on weather forecasts and hydrological forecasts (usually river discharge), and only a very few addressed drought forecasting. This is the main reason why I got interested in this particular topic: hydrometeorological drought forecasting that narrows the gap between meteorological and hydrological disciplines.

BAMS: Narrowing the gap between two disciplines apparently requires understanding of both.

SS: This work brings together the expertise of meteorology and hydrology as multifaceted, interdisciplinary research. For hydrometeorological drought forecasts, meteorologists and hydrologists have to join forces. We cannot work separately.

BAMS: What kinds of collaboration are critical in bridging this gap?

SS: In order to run the hydrological model in the forecast mode, hydrologists need meteorological forecast data, such as rainfall and temperature, as input. Meteorologists need to understand the requirements for the input data, and hydrologists need to comprehend the concepts of the meteorological forecasting systems to value the input data they use.

BAMS: What surprised you the most about the system?

SS: Henny Van Lanen and I are surprised our system could predict hydrological drought three months in advance. For instance, we showed this by providing the forecasts for July 2018, which were initiated on 2 May 2018-that was, 3 months before vast parts of Europe were hit by the severe 2018 drought.

BAMS: What was the biggest challenge you encountered in this work?

SS: The biggest challenge-besides funding to keep the system operational-was how to keep the drought forecasting system running after the European Centre for Medium Weather Forecast (ECMWF) updated their seasonal weather forecasting system and the EU Joint Research Centre (JRC) increased the domain of hydrological model LISFLOOD. 